\title{
P02-1-48 Poster session
}

\section{Protective effect of trilobatin on hydrogen peroxide-induced injury in PC12 cells}

\author{
Jianmei Gao ${ }^{1}$, Linying Feng ${ }^{2}$, Nana Chen ${ }^{1}$, Yan Deng ${ }^{2}$, Chun Lv $^{2}$ \\ ${ }^{1}$ Department of Clinical Pharmacotherapeutics, School of Pharmacy, Zunyi Medical University, China, ${ }^{2}$ Department of \\ Pharmacology, Key Laboratory of Basic Pharmacology of Ministry of Education and Joint International Research \\ Laboratory of Ethnomedicine of Ministry of Education, China
}

Oxidative stress-induced neuronal cell damage is a crucial factor in the pathogenesis of mitochondria-associated neurological diseases. Therefore, elimination of overproduction of mtROS may be a potential strategy for used in prevention and treatment of neurological diseases. In the present study, the mechanisms underlying the neuroprotective effects of trilobatin (TLB), a major bioactive compond derived from Lithocarpus polystachyus, was investigated in vitro using hydrogen peroxide-induced oxidative stress model in a neuron-like highly differentiated rat pheochromocytoma line PC12. We found that pretreatment with TLB significantly concentration-dependently suppressed hydrogen peroxideinduced PC12 cells damage by enhancing cell viability, repressing collapse of mitochondrial membrane potential and decreasing mitochondrial ROS (mtROS) generation, thereby deferring cell apoptosis. Further study demonstrated that TLB not only increased the enzymatic activities of manganese superoxide dismutase (SOD2), glutathione peroxidase (GSH-Px) and isocitrate dehydrogenase 2 (IDH2), but also activated Sirt3 within the mitochondria, then leading to improved complex I activity and ATP synthesis. Furthermore, TLB up-regulated the level of phosphorylated adenosine monophosphate-activated protein kinase (p-AMPK). Intriguingly, TLB failed to suppress hydrogen peroxide-induced cytotoxicity in the presence of the AMPK inhibitor Compond C, indicating the effects of TLB on mtROS homeostasis regulation were dependent on AMPK/Sirt3 signaling pathway. In conclusion, our findings, at the first time, demonstrated that TLB protected against oxidative injury in neuronal PC12 cells through regulating mtROS homeostasis, which, at least partly, was mediated through the reciprocity of AMPK/Sirt3 signaling pathway. 